\title{
EACH HOMOGENEOUS NONDEGENERATE CHAINABLE CONTINUUM IS A PSEUDO-ARC
}

\author{
R. H. BING ${ }^{1}$
}

The endeavor to find all homogeneous plane continua continues. The simple closed curve and the point are obvious examples. The discovery of the pseudo-arc $[1 ; 6]$ should have exploded (but did not) the conjectures that there are no others. A history of the problem with a discussion of various false starts is given in [4]. Finding the circle of pseudo-arcs [4] raised the number of known examples to four. Are there others as yet undiscovered? Jones showed [5] that each one which does not separate the plane is indecomposable. The theorem in this paper narrows the field for search still further.

We recall the following definitions:

A set $X$ is homogeneous if for each pair of points $p, q$ of $X$ there is a homeomorphism of $X$ onto itself that takes $p$ onto $q$.

A continuum is nondegenerate if it contains more than one point.

An $\epsilon$-chain is a finite ordered collection $d_{1}, d_{2}, \cdots, d_{n}$ of open sets, each of diameter less than $\epsilon$, such that $d_{i}$ intersects $d_{j}$ if and only if $i$ and $j$ are adjacent integers.

A snakelike or chainable continuum is a compact metric continuum $M$ such that for each positive number $\epsilon, M$ can be covered by an $\boldsymbol{\epsilon}$-chain.

A point $p$ is an end point of a snakelike continuum $M$ if for each positive number $\epsilon$ there is an $\epsilon$-chain covering $M$ such that the first link of the chain contains $p$.

A continuum is indecomposable if it is not the sum of two proper subcontinua. It is hereditarily indecomposable if each subcontinuum of it is indecomposable.

A pseudo-arc is a nondegenerate, hereditarily indecomposable, chainable continuum. Any two such continua are homeomorphic [2].

ThEOREM. Each homogeneous, nondegenerate, chainable continuum is a pseudo-arc.

Proof. First we show that $M$ has an end point $p$. For each integer $n$, let $q_{n}$ be a point of $M$ such that a $1 / n$-chain covers $M$ and an end link of this chain contains $q_{n}$. Some subsequence of $q_{1}, q_{2}, \cdots$ converges to a point $q$. Then the point $q$ of $M$ has the following property: 1958.

Presented to the Society, February 23, 1957; received by the editors October 16,

Work on this paper was supported by Contract NSF G1219. 
Property of $q$. For each neighborhood $N$ of $q$ and each positive number $\epsilon$ there is an $\epsilon$-chain covering $M$ one of whose end links intersects $M$ and lies in $N$. It follows from the homogeneity of $M$ and the fact that each homeomorphism of $M$ onto itself is uniformly continuous that each point of $M$ has the Property of $q$.

Let $d_{1}$ be an end link of a 1 -chain covering $M$ such that $d_{1}$ contains a point $p_{1}$ of $M$. Since $p_{1}$ has the Property of $q$, there is an end link $d_{2}$ of a $1 / 2$-chain covering $M$ such that $d_{1}$ contains $\bar{d}_{2}$ and $d_{2}$ contains a point $p_{2}$ of $M$. Also, there is an end link $d_{3}$ of a $1 / 3$-chain covering $M$ such that $d_{2}$ contains $\bar{d}_{3}$ and $d_{3}$ contains a point $p_{3}$ of $M$. Similarly, we obtain $d_{4}, d_{5}, \cdots$. Then the point $p$ which is the intersection of $d_{1}, d_{2}, \cdots$ is an end point of $M$.

Finally we show that $M$ is hereditarily indecomposable. Assume $M$ contains a continuum $H$ which is the sum of two proper subcontinua $H^{\prime}, H^{\prime \prime}$. Let $p$ be a point of $H^{\prime} \cdot H^{\prime \prime}$. Then it follows from the homogeneity of $M$ that $p$ is an end point of $M$. However, as noted in [3], this would imply that one of $H^{\prime}, H^{\prime \prime}$ contains the other and this is impossible since each is a proper subcontinuum of their sum.

\section{REFERENCES}

1. R. H. Bing, A homogeneous indecomposable plane continuum, Duke Math. J. vol. 15 (1948) pp. 729-742.

2. - Concerning hereditarily indecomposable continua, Pacific J. Math. vol. 1 (1951) pp. 43-51.

3. - , Snake-like continua, Duke Math. J. vol. 18 (1951) pp. 653-663.

4. R. H. Bing and F. B. Jones, Another homogeneous plane continuum, Trans. Amer. Math. Soc. vol. 90 (1959) pp. 171-192.

5. F. B. Jones, Certain homogeneous unicoherent indecomposable continua, Proc. Amer. Math. Soc. vol. 2 (1951) pp. 855-859.

6. E. E. Moise, $A$ note on the pseudo-arc, Trans. Amer. Math. Soc. vol. 64 (1949) pp. 57-58.

UNIVERSITY OF WisconsIN 